\title{
Cleistogamia em Ruellia menthoides (Nees) Hiern e R. brevifolia (Pohl) C. Ezcurra (Acanthaceae) em fragmento florestal do Sudeste brasileiro ${ }^{1}$
}

\author{
Natália A. de Souza Lima², Milene Faria Vieira ${ }^{2,3}$ Rita Maria de Carvalho-Okano² e Aristéa Alves Azevedo ${ }^{2}$
}

Recebido em 08/06/2004. Aceito em 11/11/2004

\begin{abstract}
RESUMO - (Cleistogamia em Ruellia menthoides (Nees) Hiern e R. brevifolia (Pohl) C. Ezcurra (Acanthaceae) em fragmento florestal do Sudeste brasileiro). São abordados a morfologia e a biologia floral de Ruellia menthoides (Nees) Hiern e R. brevifolia (Pohl) C. Ezcurra, na Reserva Florestal Mata do Paraíso, remanescente de Floresta Atlântica, em Viçosa, Zona da Mata de Minas Gerais, Brasil. Ruellia menthoides e $R$. brevifolia são espécies cleistógamas, ou seja, apresentam dimorfismo floral, produzindo flores casmógamas normais (CA) e cleistógamas (CL). Além disso, formas intermediárias entre esses tipos florais (flores casmógamas reduzidas e semicleistógamas) também são observadas. Em $R$. menthoides a cleistogamia é inédita, ampliando o número de espécies desse gênero com esse mecanismo reprodutivo. A alogamia, nessas espécies, é favorecida pela produção de flores CA hercogâmicas, especialmente em $R$. menthoides, e pela dicogamia parcial. A floração de ambas as espécies ocorreu durante todo o ano. Houve pequena sobreposição do período de produção dos dois tipos florais em $R$. menthoides e ampla sobreposição em $R$. brevifolia. Em R. menthoides, o início da produção de flores CL coincidiu com o período de transição da estação úmida para a seca, e em $R$. brevifolia, a produção desse tipo floral ocorreu principalmente na estação seca. A complexidade taxonômica de Ruellia parece estar relacionada à escassez de informações sobre a biologia reprodutiva de suas espécies, que é agravada com o polimorfismo floral das espécies cleistógamas.
\end{abstract}

Palavras-chave: cleistogamia, Floresta Atlântica, morfologia floral, polimorfismo floral, Ruellia

\begin{abstract}
Cleistogamy in Ruellia menthoides (Nees) Hiern and R. brevifolia (Pohl) C. Ezcurra (Acanthaceae) in a forest fragment of Southeastern Brazilian). Floral morphology and biology of Ruellia menthoides (Nees) Hiern and R. brevifolia (Pohl) C. Ezcurra were analyzed in Reserva Florestal Mata do Paraíso, a remainder of the Atlantic forest, in Viçosa, Zona da Mata, Minas Gerais State, Brazil. $R$. menthoides and $R$. brevifolia are cleistogamous, e.g., they show floral dimorphism, with normal chasmogamous (CA) and cleistogamous (CL) flowers. Intermediate forms between these two floral types (miniature chasmogamous and semicleistogamous flowers) are also observed. $R$. menthoides cleistogamy is a novel discovery, which augments the number of species of this genus with that reproductive system. In these species, alogamy is favored by CA hercogamic flower production, verified mainly in R. menthoides, and by partial dichogamy. In both species, flowers production occurs throughout the year. There was a small production period overlap of both types of flowers in $R$. menthoides and a large overlap in $R$. brevifolia. The initial production of CL flowers in $R$. menthoides coincided with the transition period from the wet to the dry season and in $R$. brevifolia, such type of flowers was produced mainly in the dry season. The taxonomic complexity of Ruellia may be related to the scarcity of information on the reproductive biology of its species, and by the floral polymorphism of the cleistogamous species.
\end{abstract}

Key words: cleistogamy, Atlantic forest, floral morphology, floral polymorphism, Ruellia

\section{Introdução}

A família Acanthaceae consiste de cerca de 229 gêneros e 3.450 espécies predominantemente tropicais (Mabberley 1997). O gênero Ruellia, um dos maiores da família, compreende aproximadamente 250 espécies, amplamente distribuídas nas regiões tropicais e subtropicais, especialmente no Novo Mundo, seu principal centro de diversidade (Ezcurra 1989; 1993).

Estudos sobre a biologia reprodutiva em espécies de Ruellia são relativamente escassos. Dentre as espécies estudadas a autocompatibilidade (Long 1964; 1966; 1971; 1974; Machado \& Sazima 1995; Piovano et al. 1995; Braz et al. 2000; N.A.S. Lima, dados não publicados; Lima et al., dados não publicados; Sigrist \& Sazima 2002), incluindo a cleistogamia, tem sido registrada.

A cleistogamia é amplamente distribuída entre as angiospermas, ocorrendo em pelo menos 287 espécies pertencentes a 56 famílias (Lord 1981). Sua importância na reprodução em espécies de Acanthaceae, especialmente de Ruellia, parece ser equivalente ao

\footnotetext{
Parte da Dissertação do Mestrado da primeira Autora

2 Departamento de Biologia Vegetal, Universidade Federal de Viçosa, CEP 36570-000, Viçosa, MG, Brasil

3 Autor para correspondência: mfvieira@ufv.br
} 
papel que esse mecanismo exerce na reprodução de espécies de Poaceae e Orchidaceae (Long 1971). Em espécies desse gênero, verificam-se, além de flores cleistógamas, flores casmógamas e formas intermediárias entre esses dois tipos florais, ou seja, flores casmógamas reduzidas e semicleistógamas (Long 1971; 1974). Esse polimorfismo floral pode indicar grande plasticidade das espécies (Ezcurra 1993) e, provavelmente, o variado sistema reprodutivo dessas plantas associado à escassez de informações sobre esse aspecto, seja uma das causas da complexidade taxonômica do grupo (Long \& Uttal 1962; Long 1971; 1974; 1975).

O presente estudo teve como objetivo analisar a morfologia e a biologia floral de Ruellia menthoides e $R$. brevifolia, visando contribuir com informações que possam auxiliar na compreensão da diversidade da morfologia floral dessas espécies.

\section{Material e métodos}

Local de estudo - O trabalho foi realizado na Reserva Florestal Mata do Paraíso. Essa Reserva consiste de uma área com aproximadamente 194 ha localizada no município de Viçosa (2048'07”S e 4251'31'W), Zona da Mata de Minas Gerais. A região pertence aos domínios da Floresta Atlântica, sendo classificada por Veloso et al. (1991) como Floresta Estacional Semidecidual Submontana. No século XIX, a área dessa reserva foi desmatada, tendo ocorrido intensa retirada de madeira. Posteriormente, foi utilizada para cultivos, principalmente de café, e uso de pastagens (N. Leal-Filho, dados não publicados). Hoje, a mata existente é de regeneração secundária (P.S. Castro, dados não publicados). O clima da região caracteriza-se por verões quentes e úmidos e invernos frios e secos (N. Leal-Filho, dados não publicados). A média pluviométrica nos anos de 1991 a 1999 foi de $1.248,7 \mathrm{~mm} /$ ano (F.J. Soares Júnior, dados não publicados). Segundo esse autor, a temperatura média nesses anos foi de $20^{\circ} \mathrm{C}$ e a umidade relativa do ar de aproximadamente $81 \%$.

Para estudos complementares, indivíduos de $R$. menthoides e $R$. brevifolia, oriundos da Reserva, foram cultivados no Horto Botânico do Departamento de Biologia Vegetal da Universidade Federal de Viçosa.

Espécies estudadas - Ruellia menthoides é herbácea, possui caule quadrangular, ereto ou decumbente, com 0,5 a $0,8 \mathrm{~m}$ alt. Suas folhas são opostas, subsésseis e elípticas e as inflorescências são axilares com flores brancas. Essa espécie é restrita ao território nacional, registrada nos Estados do Rio de Janeiro, Mato Grosso, Amazonas (Nees 1847), Minas Gerais e São Paulo (Braz et al. 2002). Na Reserva, foi principalmente observada em locais abertos a parcialmente sombreados, na margem da trilha principal. O materialtestemunho está depositado no acervo do Herbário VIC (n. 23.745) Universidade Federal de Viçosa.

Ruellia brevifolia é herbácea, possui caule ligeiramente anguloso, ereto com cerca de $1 \mathrm{~m}$ alt. Suas folhas são opostas, pecioladas e ovadas e as inflorescências são axilares com flores vermelhas. Essa espécie é amplamente distribuída na América do Sul, ocorrendo desde a Colômbia até o norte da Argentina (Ezcurra 1993). No Brasil, R. brevifolia foi registrada nos Estados de Santa Catarina (Wasshausen \& Smith 1969), São Paulo (Kameyama 1991) e Minas Gerais (Kameyama 1995; Braz et al. 2002). Na Reserva, foi encontrada principalmente em locais abertos a parcialmente sombreados, na margem da trilha principal, distante cerca de $1 \mathrm{~km}$ do local de ocorrência de $R$. menthoides. Material-testemunho está depositado no acervo do Herbário VIC (n. 24.910).

Morfologia e biologia floral - Durante o trabalho de campo, realizado de setembro/1999 a fevereiro/2001, foram registrados, para cada espécie, a forma, a coloração e o tamanho da corola, e a posição relativa das anteras e estigma das flores casmógamas (CA) e cleistógamas (CL). Desenhos de flores frescas ou previamente estocadas em etanol $70 \%$ foram confeccionados com auxílio de microscópio estereoscópico. A presença dos tipos florais foi acompanhada, semanalmente, de fevereiro/2000 a janeiro/2001, principalmente pela manhã.

Em flores CA, foram registrados o período de antese, sua duração, a receptividade do estigma, a deiscência das anteras e a presença de néctar. A receptividade do estigma foi testada em diferentes horários do dia utilizando-se peróxido de hidrogênio 3\% (Dafni 1992). A presença do néctar foi verificada utilizando-se glico-fita (da Eli Lilly do Brasil Ltda), que testa a presença de glicose, um dos principais açúcares encontrados no néctar (Baker \& Baker 1983).

Em flores CL, foi verificado o local de germinação de tubos polínicos. Para tanto, flores com anteras fechadas ou recentemente abertas foram estocadas em etanol $70 \%$. Posteriormente, anteras e pistilo foram 
analisados em microscopia de fluorescência, utilizando-se a técnica de Martin (1959).

\section{Resultados e discussão}

Ruellia menthoides e R. brevifolia são, de acordo com a definição de Lord (1981), espécies cleistógamas, pois além de produzirem flores $\mathrm{CA}$, produzem flores $\mathrm{CL}$ e formas intermediárias entre esses tipos florais (Fig. 1, 2). A cleistogamia em $R$. menthoides é inédita e amplia o número de espécies desse gênero com dimorfismo floral (Tab. 1), enquanto em $R$. brevifolia confirma as observações realizadas por Piovano et al. (1995), em indivíduos cultivados na Argentina, e por Sigrist \& Sazima (2002), em indivíduos de população
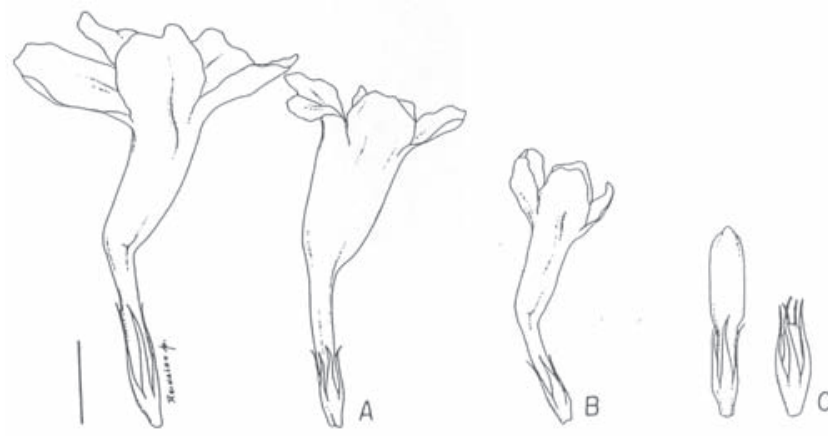

Figura 1. Flores de Ruellia menthoides (Nees) Hiern: casmógamas normais (A), casmógama reduzida (B) e cleistógamas (C). Barra $=5 \mathrm{~mm}$
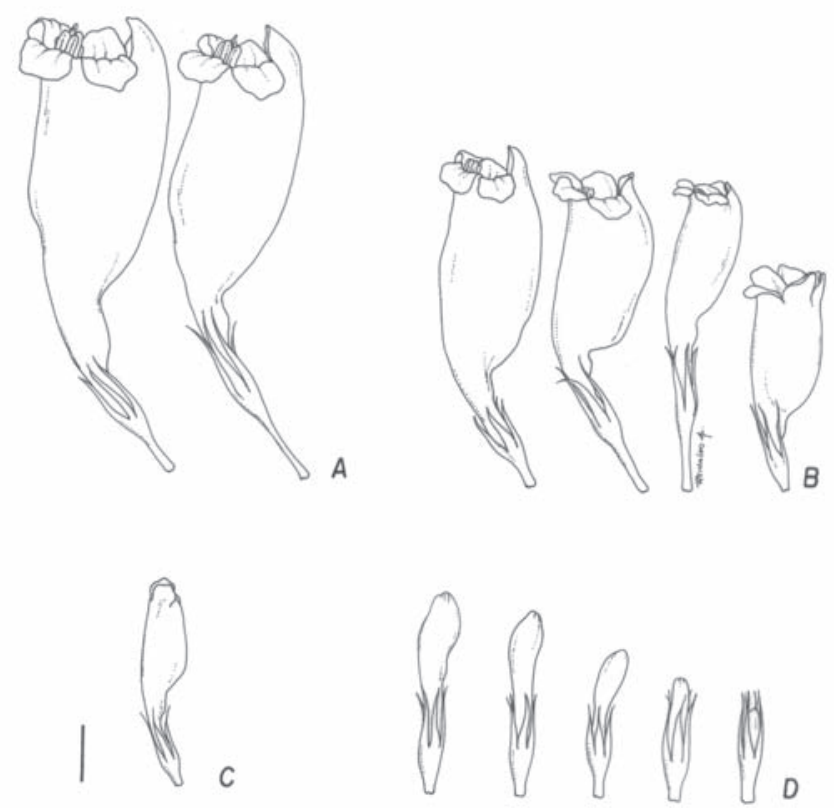

Figura 2. Flores de Ruellia brevifolia (Pohl) C. Ezcurra: casmógamas normais (A), casmógamas reduzidas (B), semicleistógama $(C)$ e cleistógamas (D). Barra $=5 \mathrm{~mm}$.
Tabela 1. Espécies cleistógamas de Ruellia (Acanthaceae).

\begin{tabular}{|c|c|}
\hline Espécies & Referências \\
\hline Ruellia brevifolia (Pohl) C. Ezcurra & $\begin{array}{l}\text { Piovano et al. (1995), } \\
\text { Sigrist \& Sazima (2002), } \\
\text { presente estudo }\end{array}$ \\
\hline R. brittoniana Leonard & Long (1977) \\
\hline R. caroliniensis (Walt.) Steud. & $\begin{array}{l}\text { Long \& Uttal (1962), Long } \\
\text { (1971) }\end{array}$ \\
\hline $\begin{array}{l}\text { R. ciliatiflora Hook. } \\
\qquad(=R \text {. lorentziana } \text { Griseb. })\end{array}$ & $\begin{array}{l}\text { Santos Biloni (1963), } \\
\text { Ezcurra (1993) }\end{array}$ \\
\hline R. coeruelea Morong & Ezcurra (1993) \\
\hline R. geminiflora Kunth & $\begin{array}{l}\text { Lima et al. (dados não } \\
\text { publicados) }\end{array}$ \\
\hline R. humilis Nutt. & Long \& Uttal (1962) \\
\hline R. lorentziana Griseb. & Sell (1977) \\
\hline $\begin{array}{l}\text { R. macrosolen Lillo } \\
\text { ex C. Ezcurra }\end{array}$ & Ezcurra (1993) \\
\hline R. menthoides (Nees) Hiern & Presente estudo \\
\hline $\begin{array}{l}\text { R. nudiflora (Engelm. \& } \\
\text { A. Gray) Urb. }\end{array}$ & Long (1977) \\
\hline $\begin{array}{l}\text { R. pedunculata Torr. ex } \\
\text { A. Gray }\end{array}$ & Long \& Uttal (1962) \\
\hline R. purshiana Fernald & Uttal (1965) \\
\hline R. strepens $\mathrm{L}$. & Long \& Uttal (1962) \\
\hline R. tuberosa L. & Long (1977) \\
\hline
\end{tabular}

natural em Campinas, São Paulo. As flores CL são importantes para assegurar a produção de sementes em situações de baixa polinização cruzada, baixa densidade de plantas, competição por polinizadores ou escassez deles (Levin 1972; Jain 1976; Macnair 1989). Em flores CA de $R$. menthoides, por exemplo, raramente foram observados visitantes florais, no local de estudo, e as suas flores CL resultaram em alta produção de frutos $(96 \%)$, com sementes viáveis (N.A.S. Lima, dados não publicados). Além disso, segundo Schemske (1978), em flores CL há redução de tecidos florais e a eliminação de gastos com a produção de néctar, diminuindo o custo energético para a planta, o que deve ser vantajoso em período de escassez hídrica, ocasião de maior produção dessas flores (Piovano et al. 1995; Sigrist \& Sazima 2002; presente estudo).

As flores CA de $R$. menthoides são sésseis, incluindo as casmógamas normais ("normal chasmogamic flower", Fig. 1A) e as reduzidas ("miniature chasmogamic flower", Fig. 1B) (sensu Long 1971) medem de 15,0-25,0 mm compr. (N=15) e possuem corola zigomorfa, infundibuliforme, branca, podendo apresentar traços arroxeados no lobo inferior; os estames são didínamos e epipétalos, inclusos. A abertura dessas flores, caracterizada pela separação e expansão dos lobos da corola, ocorre pela manhã, 
por volta das 9:30 $\mathrm{h}$; permanecem abertas durante cerca de 26 horas. Logo após a expansão da corola, as anteras apresentam-se deiscentes, enquanto a presença de néctar (confirmado com a glico-fita) foi observada ao longo do dia. O estigma posiciona-se acima das anteras ou, raramente, na mesma altura e permanece não-receptivo até cerca de quatro horas após a abertura da flor. No período da tarde e na manhã seguinte à abertura da flor, o estigma apresenta-se receptivo. $R$. menthoides apresenta características florais de espécies melitófilas (Faegri \& van der Pijl 1979), confirmadas por N.A.S. Lima (dados não publicados).

As flores CA de $R$. brevifolia, incluindo as casmógamas normais (Fig. 2A) e as reduzidas (Fig. 2B), são pediceladas, medem de 16,0-30,0 mm compr. $(\mathrm{N}=14)$, zigomorfas e possuem corola tubulosa ventricosa, externamente vermelha e internamente amarelada; os estames também são didínamos e epipétalos, subexsertos. A abertura das flores, caracterizada pela separação e expansão dos lobos da corola, inicia-se por volta das 8:00 h, mas foram observadas flores abrindo no final da manhã e à tarde. A deiscência das anteras ocorre durante a abertura da flor e a presença de néctar também foi observada ao longo do dia. Em $72 \%$ das flores amostradas $(\mathrm{N}=93)$, anteras e estigma apresentaram a mesma altura, em $23 \%$ o estigma encontrava-se acima das anteras e em $5 \%$, abaixo delas. Essa percentagem de flores hercogâmicas (28\%) difere da observada em outras localidades. Por exemplo, Sigrist \& Sazima (2002) mencionaram que $71 \%$ das flores $(\mathrm{N}=110)$, em indivíduos ocorrentes em Campinas, são hercogâmicas. Essas diferenças confirmam o polimorfismo floral das espécies de Ruellia, mencionado por Ezcurra (1993). O estigma apresentou-se receptivo à tarde, permanecendo assim até a queda da flor, ou seja, cerca de 36 horas após a abertura. As características florais de $R$. brevifolia são típicas da ornitofilia (Faegri \& van der Pijl, 1979), o que já havia sido observado por Piovano et al. (1995), Braz et al. (2000) e Sigrist \& Sazima (2002). Essas últimas autoras consideraram borboletas como polinizadores dessa espécie.

A hercogamia, observada principalmente em $R$. menthoides, e a dicogamia parcial indicada pela protandria, foram registradas por Piovano et al. (1995) em $R$. brevifolia e devem favorecer a alogamia em ambas as espécies. A hercogamia, no entanto, não deve impedir a autopolinização nas flores com estigma acima das anteras, uma vez que durante a queda da corola as anteras podem contatar o estigma. Esse processo foi observado em espécies de Ruellia, inclusive em R. brevifolia (Long 1966; 1977; Piovano et al. 1995). A variação no horário de abertura das flores, especialmente de $R$. brevifolia, e a sua duração, possibilitam que haja, concomitantemente, flores em fase masculina (doadoras de pólen) e em fase feminina (receptoras de pólen), favorecendo a alogamia.

A maioria das flores CL ("normal cleistogamic flower", sensu Long 1971) de R. menthoides é inconspícua, pois, além de apresentarem redução do tamanho da corola e do androceu (constituído de quatro pequenos estames epipétalos), comumente ficam ocultas pelo cálice (Fig. 1C). Essas flores desenvolvem-se nos nós apicais do caule e possuem corola branca, caliptrada (sensu Long 1971), medindo cerca de 4,0 mm compr. ( $\mathrm{N}=13)$. Raramente foram observadas flores CL com 9,0 mm compr. (Fig. 1C).

Em $R$. brevifolia, as flores CL são sésseis ou subsésseis, possuem corola, geralmente, branca, caliptrada e medem de 5,0-15,0 mm compr. $(\mathrm{N}=21)$ (Fig. 2D). As flores CL com corola medindo cerca de $9 \mathrm{~mm}$ podem apresentar uma tonalidade avermelhada e aquelas com 12,0-15,0 mm, raramente observadas, possuem corola vermelha e são muito semelhantes aos botões florais de flores CA. Nessa espécie foram raramente observadas flores semicleistógamas ("semicleistogamic flower", sensu Long 1971), que se caracterizam por apresentarem-se parcialmente abertas (Fig. 2C).

$\mathrm{Na}$ microscopia de fluorescência, foi observado que nas flores CL, de ambas as espécies, os grãos de pólen são depositados sobre o estigma, embora, na ocasião da deposição, alguns grãos apresentem o tubo polínico em fase inicial de desenvolvimento.

O polimorfismo floral não se restringe às espécies cleistógamas de Ruellia, ocorrendo também em espécies casmógamas, como observado em indivíduos de R. subsessilis (casmógamas normais, Fig. 3A; casmógama reduzida, Fig. 3B), na região de Viçosa. A corola dessa espécie mede de 3,0-7,0 cm compr. $(\mathrm{N}=18)$ e o tubo floral apresenta diferentes graus de curvatura (Fig. 3). Essa espécie ocorre nos Estados de Minas Gerais e Espírito Santo (Braz et al. 2002) e estudos complementares sobre a sua reprodução, em indivíduos de outras populações, poderiam esclarecer se essa ampla plasticidade floral está relacionada à ocorrência de cleistogamia. Esse polimorfismo floral, além de indicar diferentes mecanismos de reprodução, incluindo síndromes florais distintas em uma mesma espécie, parece dificultar a taxonomia de Ruellia 

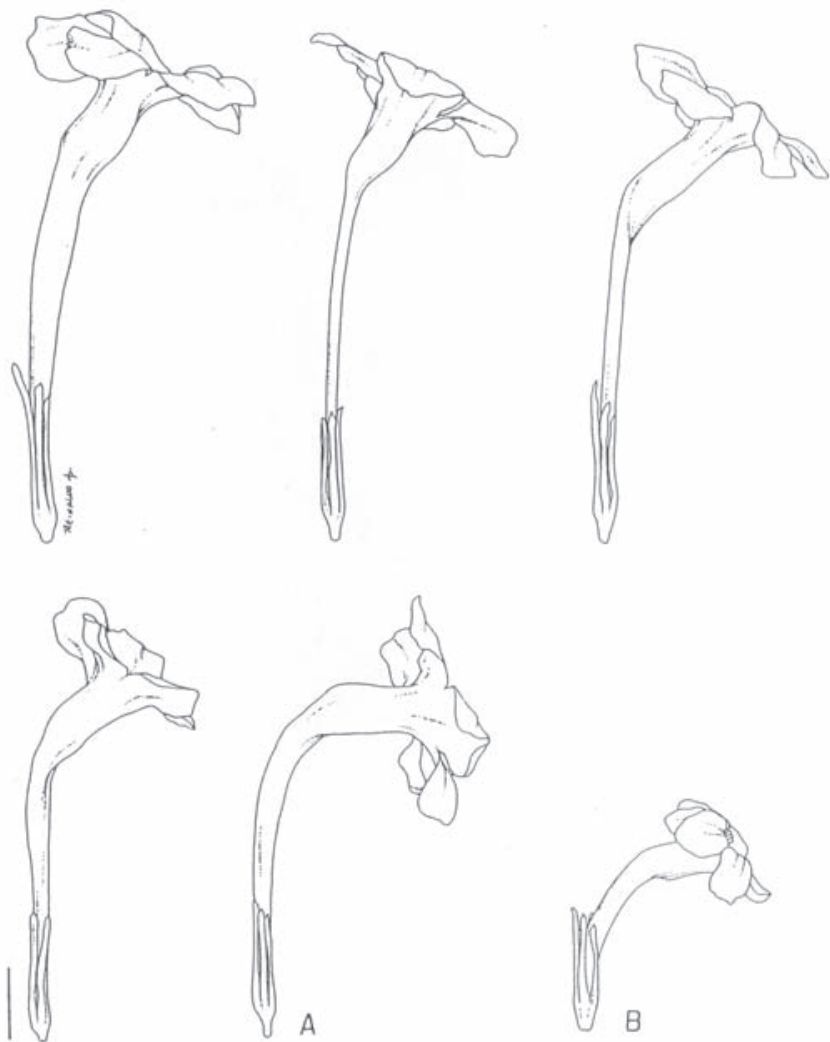

Figura 3. Flores de Ruellia subsessilis (Nees) Lindau: casmógamas normais (A) e reduzida (B). Barra $=10 \mathrm{~mm}$

(Ezcurra 1993). Braz et al. (2002), em levantamento de espécies de Acanthaceae da Reserva Florestal Mata do Paraíso, encontrou dificuldades na identificação de $R$. menthoides. Essa espécie é muito semelhante à $R$. epallocaulos Leonard ex C. Ezcurra \& Wassh. e à R. kleinii C. Ezcurra \& Wassh., ambas ocorrendo no sul do Brasil (Ezcurra 1993).

A produção de flores CA em $R$. menthoides foi verificada inicialmente em agosto, final da estação seca, e finalizou em novembro (Fig. 4), tendo sido observado expressivo aumento em setembro. As flores CL foram observadas durante quase todo o ano, exceto em outubro (Fig. 4). A maior produção de flores CL foi verificada nos meses de março e abril, período de transição entre a estação úmida e seca. Em julho e agosto, meses em que são registrados os menores índices de umidade da região, a produção de flores CL diminuiu drasticamente. Este fato, associado à produção de flores $\mathrm{CA}$ coincidindo com término da estação seca e com o aumento da temperatura e do fotoperíodo, sugere que a cleistogamia em $R$. menthoides possa ser influenciada por um ou mais fatores ambientais.

Em $R$. brevifolia, as flores CA foram observadas praticamente por todo o ano (exceto em agosto e setembro), assim como as flores CL (exceto em janeiro e fevereiro), havendo extensa sobreposição na produção desses tipos florais (Fig. 4). A maior produção de flores CL foi observada nos meses de maio a setembro, ou seja, principalmente durante a estação seca. De modo geral, um indivíduo produz, primeiramente, flores $\mathrm{CA}$, em seguida, flores $\mathrm{CA}$ e $\mathrm{CL}$ e, posteriormente, apenas flores CL. A produção de flores CL em $R$. brevifolia, principalmente no período seco, incluindo os meses de julho e agosto, e sua ausência ou baixa ocorrência durante quase todo período úmido, sugerem que as condições ambientais que podem estar influenciando a produção desse tipo floral pode ser, em parte, diferentes das que controlam sua produção em $R$. menhoides. Piovano et al. (1995) classificaram a cleistogamia em $R$. brevifolia como do tipo "ecológica" (sensu Uphof 1938), uma vez que verificaram que a seca aumentou sua ocorrência, tal como observado por Sigrist \& Sazima (2002) e pelas autoras do presente estudo.

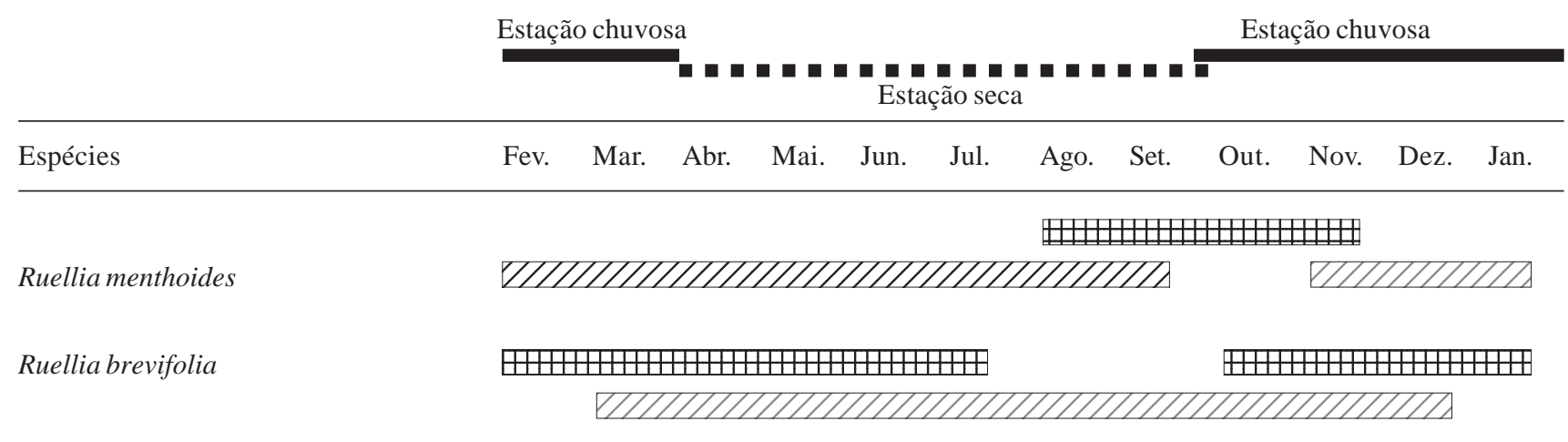

Figura 4. Fenologia de floração de espécies de Ruellia, no período de fevereiro/2000 a janeiro/2001 na Reserva Florestal Mata do Paraíso, Viçosa, MG, Brasil. $\boxplus=$ Flores casmogamas; $\boxminus=$ flores cleistógamas. 
A produção de flores CL em Ruellia tem sido relacionada a fatores ambientais (p.ex., umidade, temperatura, danos provocados por insetos a flores e frutos), genéticos e fisiológicos (Long \& Uttal 1962; Long 1971; 1974; 1977; Sell 1977; Rachuvanshi et al. 1981; Piovano et al. 1995). Goldenberg \& Shepherd (1998) sugeriram que a apomixia em Melastomataceae, mecanismo mais comumente registrado em espécies da tribo Miconeae, seja determinada filogeneticamente. De modo similar, é possível que a cleistogamia em Acanthaceae, especialmente no gênero Ruellia dado o elevado número de espécies com esse mecanismo reprodutivo, seja assim determinada. Entretanto, estudos posteriores que analisem os fatores ambientais ou fisiológicos que possam estar relacionados à produção de flores CL, nesse gênero, são necessários. A ampliação de estudos sobre os sistemas reprodutivos em espécies de Ruellia, em diferentes localidades, contribuirão para uma delimitação mais precisa desses táxons, reduzindo, provavelmente, o número de espécies desse gênero.

\section{Agradecimentos}

À CAPES, pela bolsa concedida à primeira autora; ao $\mathrm{CNPq}$, pela bolsa de pesquisa concedida à segunda autora; a Reinaldo Antônio Pinto, pela confecção das figuras.

\section{Referências bibliográficas}

Baker, H.G. \& Baker, I. 1983. Floral nectar sugar constituents in relation to pollinator type. In: C.E. Jones \& R.J. Little (eds.). Handbook of Experimental Pollination Biology. New York, Van Nostrand Reinhold Company Inc.

Braz, D.M.; Carvalho-Okano, R.M. \& Kameyama, C. 2002. Acanthaceae da Reserva Florestal Mata do Paraíso, Viçosa, Minas Gerais. Revista Brasileira de Botânica 25: 495-504.

Braz, D.M.; Vieira, M.F. \& Carvalho-Okano, R.M. 2000. Aspectos reprodutivos de espécies de Acanthaceae Juss. de um fragmento florestal do município de Viçosa, Minas Gerais. Revista Ceres 47: 229-239.

Dafni, A. 1992. Pollination ecology - A pratical approach. Oxford, Oxford University Press.

Ezcurra, C. 1989. Ruellia sanguinea (Acanthaceae) y espécies relacionadas en Argentina, Uruguay y sur de Brasil. Darwiniana 29: 269-287.

Ezcurra, C. 1993. Systematics of Ruellia (Acanthaceae) in Southern South America. Annals of the Missouri Botanical Garden 80: 784-845.

Faegri, K. \& van der Pijl, L. 1979. Principles of pollination ecology. Oxford, Pergamon Press.
Goldenberg, R. \& Shepherd, G.J. 1998. Studies on the reproductive biology of Melastomataceae in "cerrado" vegetation. Plant Systematics and Evolution 211: 13-29.

Jain, S.K. 1976. The evolution of inbreeding in plants. Annual Review of Ecology and Systematics 7: 469-495.

Kameyama, C. 1991. Acanthaceae. In: M.M.R. Melo; F. Barros; M.G.L. Wanderley; M. Kirizawa; S.L. JungMendaçolli \& S.A.C. Chiea (eds.). Flora fanerogâmica da Ilha do Cardoso. São Paulo, Instituto de Botânica.

Kameyama, C. 1995. Flora da Serra do Cipó, Minas Gerais: Acanthaceae. Boletim da Universidade São Paulo 14: $1-238$.

Levin, D.A. 1972. Competition for pollinator service: a stimulus for the evolution of autogamy. Evolution 26: 668-669.

Long, R.W. 1964. Biosystematics investigations in South Florida populations of Ruellia (Acanthaceae). American Journal of Botany 51: 842-852.

Long, R.W. 1966. Artificial inter-specific hybridization in Ruellia (Acanthaceae). American Journal of Botany 53: 917-927.

Long, R.W. 1971. Floral polymorphy and amphimitic breeding systems in Ruellia caroliniensis (Acanthaceae). American Journal of Botany 58: 525-531.

Long, R.W. 1974. Variation in natural populations of Ruellia caroliniensis (Acanthaceae). Bulletin of the Torrey Botanical Club 101: 1-6.

Long, R.W. 1975. Artificial interspecific hybridization in temperate and tropical species of Ruellia (Acanthaceae). Brittonia 27: 289-296.

Long, R.W. 1977. Artificial induction of obligate cleistogamy in species-hibrids in Ruellia (Acanthaceae). Bulletin of the Torrey Botanical Club 104: 53-56.

Long, R.W. \& Uttal, L.J. 1962. Some observations on flowering in Ruellia (Acanthaceae). Rhodora 64: 200-206.

Lord, E.M. 1981. Cleistogamy: a tool for the study of floral morphogenesis, function and evolution. Botanical Review 47: 421-449.

Mabberley, D.J. 1997. The plant-book. A portable dictionary of vascular plants. Cambridge, Cambridge University Press.

Machado, I.C. \& Sazima, M. 1995. Biologia da polinização e pilhagem por beija-flores em Ruellia asperula Lindau (Acanthaceae) na caatinga, nordeste brasileiro. Revista Brasileira de Botânica 18: 27-33.

Macnair M.R. 1989. The potential for rapid speciation in plants. Genome 31: 203-210.

Martin, F.W. 1959. Staining and observing pollen tubes in the style by means fluorescence. Stain Technology 34: 125-128.

Nees, C.G. 1847. Acanthaceae. In: K.P. Von Martius; A.G. Eichler \& I. Urban (eds.). Flora Brasiliensis 9: 1-164. F. Fleischer, Lipsiae.

Piovano, M.A.; Galeto, L. \& Bernardello, L.M. 1995. Floral morphology, nectar features and breeding system in Ruellia brevifolia (Acanthaceae). Revista Brasileira de Biologia 55: 409-418. 
Rachuvanshi, S.S.; Pathak, C.S. \& Singh, R.R. 1981 Gibberellic acid response and induced chasmogamous variant in cleistogamous Ruellia hybrid (R. tweediana X R. tuberosa). Botanical Gazette 142: 40-42.

Santos Biloni, J. 1963. Observaciones sobre la floración de la Acantácea indígena Ruellia lorentziana Grisebach. Darwiniana 12: 661-663.

Schemske, D.W. 1978. Evolution of reproductive characteristics in Impatiens (Balsaminaceae): the significance of cleistogamy and chasmogamy. Ecology 59: 596-613.

Sell, Y. 1977. La cleistogamie chez Ruellia lorentziana Griseb. et quelques autres Acanthacees. Bericht der Deutschen botanischen Gesellschaft 90: 135-147.
Sigrist, M.R. \& Sazima, M. 2002. Ruellia brevifolia (Pohl) Ezcurra (Acanthaceae): fenologia da floração, biologia da polinização e reprodução. Revista Brasileira de Botânica 25: 35-42.

Uphof, J.C.Th. 1938. Cleistogamic flowers. Botanical Review 4: 21-49.

Uttal, L.J. 1965. Observations on Ruellia purschiana (Acanthaceae) in Virginia. Castanea 30: 228-230.

Veloso, H.P.; Rangel-Filho, A.L.R. \& Lima, J.C.A. 1991. Classificação da vegetação brasileira adaptada a um sistema universal. Rio de Janeiro, Fundação Instituto Brasileiro de Geografia e Estatística - IBGE.

Wasshausen, D.C. \& Smith, L.B. 1969. Acantáceas. In: R. Reitz (ed.). Flora ilustrada Catarinense. Santa Catarina, Herbário Barbosa Rodrigues. 